\title{
Awareness about Signs of Anaemia Complicating Pregnancy
}

\author{
Sivarama Krishnan S. ${ }^{1}$, N. Hepzibah Kirubamani ${ }^{2}$ \\ ${ }^{1}$ Saveetha Medical College and Hospital, Kuthambakkam, Tamilnadu, India. ${ }^{2}$ Department of Obstetrics and \\ Gynaecology, Saveetha Medical College and Hospital, Kuthambakkam, Tamilnadu, India.
}

\section{ABSTRACT}

\section{BACKGROUND}

Iron deficiency anaemia is the most common form of anaemia and the most common nutritional disorder in India. Commonest anaemia during pregnancy is iron deficiency and it can be dual, both iron and folic acid deficiency. Women with mild anaemia often tend to be asymptomatic and anaemia is detected only on screening. Anaemia is found to be a direct contributor of postpartum haemorrhage (PPH) which is the leading cause (20\%) of maternal deaths. We wanted to assess the awareness about signs of anaemia complicating pregnancy, and its complications.

\section{METHODS}

This is a prospective cross-sectional study done among the pregnant women attending obstetrics and gynaecology department, Saveetha Medical College and Hospital. Data was collected through semi-structured questionnaire-based interview after informed consent and ethical clearance and was then analysed.

\section{RESULTS}

Hundred pregnant women were recruited for the study. Fifty percent (50\%) had satisfactory knowledge, forty six percent (46\%) had poor knowledge, and four percent $(4 \%)$ had good knowledge about anaemia during pregnancy. Out of forty six percent $(46 \%)$ of the antenatal women who had poor knowledge about anaemia during pregnancy, thirty one percent (31\%) belonged to lower socioeconomic class and the remaining fifteen percent $(15 \%)$ belonged to middle class. Fifty percent $(50 \%)$ of the antenatal women who had satisfactory knowledge about anaemia during pregnancy belonged to the middle class. Four percent (4\%) of antenatal women who had good Knowledge about anaemia during pregnancy belonged to upper socioeconomic class. Out of forty six percent (46\%) of the antenatal women who had poor knowledge about anaemia during pregnancy, ten percent $(10 \%)$ had no formal education,eighteen percent $(18 \%)$ had completed their primary school education and the remaining eighteen percent (18\%) had completed their secondary school education. Out of fifty percent(50\%) of the antenatal women who had satisfactory knowledge about anaemia during pregnancy, twenty seven percent $(27 \%)$ had completed their secondary school education and the remaining twenty three percent $(23 \%)$ were degree holders.The remaining four percent $(4 \%)$ of the antenatal women who had good knowledge about anaemia during pregnancy were degree holders.

\section{CONCLUSIONS}

Pregnant women were not aware about the grave complications of anaemia during pregnancy. Awareness must be re-emphasized through media-radio, television so that more knowledge to the general population regarding the complications of anaemia during pregnancy can be imparted and the need for regular follow up during pregnancy for decreasing the maternal mortality can be emphasized.

\section{KEY WORDS}

Pregnancy, Anaemia, Cardiac Failure, Iron Deficiency, Awareness
Corresponding Author: Dr. N. Hepzibah Kirubamani, Saveetha Medical College and Hospital, Kuthambakkam, Tamilnadu, India.

E-mail: hepsi1002@yahoo.co.in

DOI: $10.14260 / j e m d s / 2019 / 694$

Financial or Other Competing Interests: None.

How to Cite This Article:

Krishnan SS, Kirubamani NH. Awareness about signs of anaemia complicating pregnancy. J. Evolution Med. Dent. Sci. 2019;8(43):3201-3205, $10.14260 /$ jemds/2019/694

Submission 04-07-2019,

Peer Review 08-10-2019,

Acceptance 15-10-2019,

Published 28-10-2019. 


\section{BACKGROUND}

Anaemia in pregnancy is a major public health problem in India as it is a direct cause for twenty percent (20\%) maternal deaths. There is increased iron requirement during pregnancy for foetus, placenta, for RBC expansions, because of blood loss during pregnancy.[1] Pregnant women suffering from anaemia, mainly women with severe anaemia also possess the risk of increased maternal morbidity and mortality. In addition to maternal complications there exists foetal complications including foetal anaemia, low birth weight (LBW), preterm delivery, intrauterine growth restriction and perinatal Mortality.[2] The cause of anaemia during pregnancy may be due to various factors. Iron and folate deficiency, vitamin deficiencies like $A$ and $B_{12}$ as well as intestinal parasitic infections, malaria and any chronic illness have all been shown to be the major causes of anaemia during pregnancy.[3] Anaemia may range from mild, moderate to severe and the WHO pegs the haemoglobin level for each of these types of anaemia in pregnancy at 10.0-10.9 g/dl (mild anaemia) 7-9.9 g/dl (moderate anaemia) and $<7 \mathrm{~g} / \mathrm{dl}$ (severe anaemia).[4]

The unavailability of proper nutritious food, food taboos, eating and cooking customs all play a role. Symptoms may be easy fatigue, breathlessness, palpitations, pedal oedema and the signs are pallor, glossitis, tachycardia, haemic murmur and may result in CCF.[5] Effect of anaemia during pregnancy on mother are pre-eclampsia, abruptio placenta, pre-term labour and on foetus are IUGR, IUD, prematurity, LBW.[6] Anaemia is a common complication of pregnancy. Uncorrected anaemia is associated with increased incidence of premature births, perinatal mortality and significant maternal death. [7] One of the primary aims of antenatal care is prevention and treatment of anaemia during pregnancy, since the safety of labour and the puerperal state and also the future health of the patient, depend upon the state of the patient's haematological reserve. Antenatal care must also include both nutrition and education components. Pregnant mothers must be encouraged to actively participate in income generating activities in order to improve their economic and nutritional status. ${ }^{[8]}$

Treatment: Ferrous sulphate $325 \mathrm{mg}$ orally once a day is usually prescribed. One 325-mg ferrous sulphate tablet taken midmorning is usually effective. However, a Higher dose or more frequent doses causes GI adverse effects, especially constipation, and one dose blocks absorption of the next dose, hence reducing the percentage intake.[9]

Prevention: About 20\% of pregnant women do not respond to supplemental oral iron; such patients require parenteral therapy, usually iron dextran $100 \mathrm{mg}$ IM every other day for a total of $\geq 1000 \mathrm{mg}$ given over 3 weeks. $\mathrm{Hb}$ is measured weekly to determine the response to the parenteral therapy. If iron supplements are ineffective, then concomitant folate deficiency should also be suspected. Neonates born to mothers with iron deficiency anaemia usually have a normal HCT but have decreased total iron stores and hence they require early dietary iron supplements.[9]

\section{METHODS}

A prospective, cross sectional study conducted in Saveetha Medical College and Hospital, Chennai, obstetrics and gynaecology Department. Hundred pregnant women were recruited. After informed consent and ethical clearance, the tools used for this study was interview with semi structured questionnaire of thirty-two items. Data about sociodemographic details, awareness about knowledge, attitude and practice were collected about anaemia complicating pregnancy, its complications and management were collected and analysed. The sample size of the research is 100. Convenient sampling technique was used for this study for calculating the sample size. Validated semi structure questionnaire was used to collect the data for this study.

\section{Inclusion Criteria}

100 antenatal mothers who were under the age group of 23 to 35 years were included in the study after their informed consent was obtained

\section{Exclusion Criteria}

The people who had language problems, and patients who were not willing to take part in the study was excluded.

\section{Statistical Analysis}

Convenient sampling technique was done here. First 100 antenatal mothers who were comfortable and gave informed consent, were asked knowledge, practice, attitude questions and were taken into this study.

\section{RESULTS}

\section{Age}

The study sample consisted of hundred antenatal mothers, out of which twenty eight (28\%) of women were 20-25 years old, forty percent $(40 \%)$ of women were $25-30$ years old, twenty one percent (21\%) of women were 30-35 years old and the remaining eleven percent $(11 \%)$ of women were of age above 35 years.

\section{Distribution of Gravida}

Out of hundred pregnant women who participated in this study, thirty nine percent (39\%) of the women were gravida 1 (primi gravida).fifty two percent (52\%) of women were gravida 2 (G2P1), out of which twenty three percent (23\%) of women are (G2P0A1) wherein it is their second pregnancy and they have had an abortion before. Nine percent (9\%) of women are gravida 3 , in which four percent (4\%) of women have one (1) live birth and one (1) abortion (G3P1A1).

\section{Socioeconomic Group Analysis}

Out of the hundred antenatal mothers participated in the study, thirty one percent (31\%) of women belonged to lower socioeconomic class, sixty five percent $(65 \%)$ belonged to middle class and the remaining four percent (4\%) of women belonged to upper socioeconomic class. 

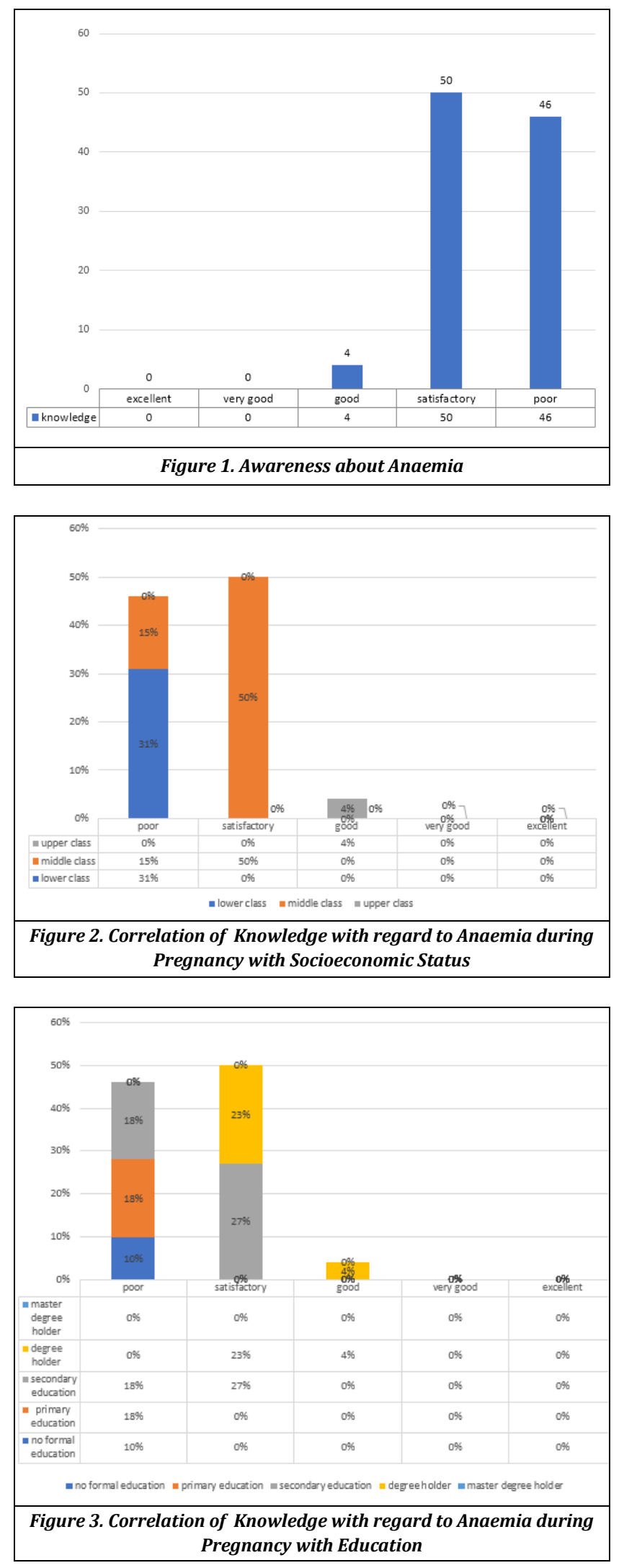

\section{Education Status}

Out of hundred antenatal women who participated in the study,ten percent $(10 \%)$ were no formal education,eighteen percent $(18 \%)$ had completed their primary school education,forty five percent (45\%) had completed their secondary school education and twenty seven percent (27\%) were degree holders.

\section{Awareness about Anaemia}

Fig. 1 depicts the distribution of knowledge among hundred in a bar graph. Most of the antenatal mother's fifty percent (50\%) had satisfactory knowledge, forty-six percent (46\%) had poor knowledge, and four percent (4\%) had good knowledge about anaemia during pregnancy.

\section{Practice}

All the antenatal mothers (100\%) had taken iron supplements regularly and felt healthy. Thirty percent (30\%) of the antenatal mother took native treatment along with iron tablets. Eighty percent $(80 \%)$ of them opted to visit the doctor when found anaemic

\section{Correlation of Knowledge with Socioeconomic Status}

Out of the forty six percent (46\%) of the antenatal women who had poor knowledge about anaemia during pregnancy, thirty one percent (31\%) belonged to lower socioeconomic class and the remaining fifteen percent $(15 \%)$ belonged to middle class. The fifty percent (50\%) of the antenatal women who had satisfactory knowledge about anaemia during pregnancy, all of them belonged to middle class. The four percent (4\%) of the antenatal women who had good Knowledge about anaemia during pregnancy, all of them belonged to upper socioeconomic class. Out of the hundred women who participated in the study nobody had very good or excellent knowledge about anaemia during pregnancy.

\section{Correlation of Knowledge with Education}

Out of forty six percent (46\%) of the antenatal women who had poor knowledge about anaemia during pregnancy, ten percent $(10 \%)$ had no formal education,eighteen percent (18\%) had completed their primary school education and the remaining eighteen percent $(18 \%)$ had completed their secondary school education. Out of fifty percent (50\%) of the antenatal women who had satisfactory knowledge about anaemia during pregnancy, twenty seven percent $(27 \%)$ had completed their secondary school education and the remaining twenty percent $(23 \%)$ were degree holders. The remaining four percent $(4 \%)$ of the antenatal women who had good knowledge about anaemia during pregnancy were degre holders.

\section{DISCUSSION}

Anaemia is widespread and it is a major public health problem associated with increased risk of morbidity and mortality especially among pregnant women.[10] In the study, knowledge among pregnant and reproductive women regarding anaemia during pregnancy, the result revealed that majority of women fifty percent (50\%) had satisfactory knowledge on anaemia during pregnancy, forty six percent (46\%) had poor knowledge, and four percent (4\%) had good knowledge. The mean percentage of knowledge of antenatal mothers about anaemia during pregnancy was $29.9 \%$.

A similar study had been conducted on maternal knowledge and practices related to anaemia and iron supplementation in Rural Malawi in 2009.[11] The findings show that the knowledge of the study sample was as follows: Majority of the women (96.6\%) knew about anaemia and many of them correctly indicated the causes, signs and 
symptoms and also about the prevention and treatment of anaemia.

A similar study had been conducted on Awareness of Anaemia among Pregnant mothers and the impact of Demographic Factors on their Haemoglobin Status in Government Theni Medical College, Tamil Nadu, India.[12] The study included 600 antenatal women belonging to different gestational age. They were interviewed about their knowledge regarding anaemia, out of which $76.5 \%$ are aware of anaemia. Only forty seven percent $(47 \%)$ of the study population knows that anaemia is more common among pregnant women. Majority of women knows about the complications of anaemia (53.5\%) and the role of iron therapy $(75.5 \%)$. In the study population, fifty one percent (51\%) had taken iron tablets regularly, thirty two percent (32\%) had irregular intake, and seventeen percent $117 \%)$ had not taken iron supplementation.

A similar study had been conducted on the impact of maternal education and socioeconomic status on maternal nutritional knowledge and practices regarding iron-rich foods and iron supplements in Saudi Arabia in 2011. The finding shows that the demographic characteristics of the study sample were influenced the knowledge of mothers as follows: twenty two percent (22\%) of the women were of no formal education, while twenty five percent $(25 \%)$ of the women had passed the 10th grade, and twelfth percentage $(12 \%)$ had completed master's degree. About twelfth percentage $(12 \%)$ had a monthly income Rs. $5000-10000$ per month, while thirty-eight (38\%) earning between Rs. 1000020000 per month and seventeen percentage $(17 \%)$ were earning >Rs. 20000 per month. About twenty nine percent (29\%) of the women were primi gravida, eighteen percent $(18 \%)$ had one child, and twenty two percent $(22 \%)$ had 2 children. However, significant relationship between educational status and knowledge regarding iron-rich foods was noticed. Higher level of knowledge was observed in subjects with higher educational background compared to non-formal education women regarding iron-rich foods. The prevalence of anaemia in pregnant women is $55.9 \%$ worldwide and varies between thirty-five percent (35\%) and hundred percent in developing countries.[13]

A study had been conducted on anaemia awareness, beliefs and practices among pregnant women: A baseline assessment at Brosankro community in Ghana. The study included pregnant women belonging to reproductive age group and on average, they have three successful births with normal birth intervals. Respondents largely showed high anaemia awareness levels by attempting to: define the disease in their laypersons' understanding; giving some symptoms regarding it; and also identifying some of the causes of anaemia. Few had no idea about the causes and effects of anaemia. Poor diet was found to the most common cause of anaemia followed by malaria, then worm infestations and others. Contrary to the high consciousness that poor diet is the number one cause of anaemia in pregnancy, as there is only a limited knowledge of iron-rich food sources that can help in preventing anaemia. Also, among the minority who claim knowledge of food sources against anaemia, less than half of them eat such food during pregnancy. Cultural and religious beliefs on food restrictions exist among the pregnant women. Such beliefs deprive women of essential nutrients including iron to be taken through diets. Meanwhile, most of the women have not taken their intermittent preventive treatment (IPT). These women also depend on unimproved drinking water sources coupled with ineffective hand washing practices (i.e. washing with soap or soap alternatives). On top of that, most respondents have not taken any deworming measures, predisposing them to infections from poor water sources and ineffective handwashing practices. There are therefore health risks (Including anaemia) among the pregnant women given the existing practices since barriers to malnutrition (knowing and eating nutritious food); and parasitic infections like malaria and worms (Especially hookworm) via use of ITBNs, IPTs, deworming, improved drinking water sources and effective hand washing are compromised. Though anaemia awareness levels are high, the women's understanding on the barriers that can be used to prevent anaemia has to be deepened. Continuous education and sensitization on locally available and low-cost nutritious food, avoiding parasitic infections, and discouraging undue food restrictions from beliefs is highly recommended to improve the nutritional status of pregnant women.[14]

\section{CONCLUSIONS}

This study points out the lack of knowledge among antenatal mothers regarding anaemia and its complications. Participants were not aware of the grave complications of anaemia like heart failure, postpartum haemorrhage and increased maternal mortality. Anaemia contributes to increased maternal mortality and morbidity because of its complications during pregnancy. It is the need of the hour to impart basic knowledge about anaemia and its complications which can have a major impact on our health care system. The important initial step is creating awareness, among low socio-economic societies about the symptoms of anaemia and its complications as they are more prone to the disease. By improving awareness, women will approach doctors early and thereby maternal mortality and morbidity rates can be reduced. More awareness programmes are recommended for women of reproductive age group.

\section{ACKNOWLEDGEMENT}

I would like to thank the Director of Saveetha Medical College and Hospital, Prof. Saveetha Rajesh for the support and encouragement, Dean of Saveetha Medical College and Hospital Dr. J. Damodaran for permitting me to conduct the study and for guiding me. I would like to thank the participants of the study.

\section{REFERENCES}

[1] Allen LH. Anemia and iron deficiency: effects on pregnancy outcome. Am J Clin Nutr 2000;71(5):1280S$4 \mathrm{~S}$. 
[2] Haggaz AD, Radi EA, Adam I. Anaemia and low birth weight in western Sudan. Trans R Soc Trop Med Hyg 2010;104(3):234-6.

[3] Uneke CJ, Duhlinska DD, Igbinedion EB. Prevalence and public-health significance of HIV infection and anaemia among pregnant women attending antenatal clinics in south-eastern Nigeria. J Health Popul Nutr 2007;25(3):328-35.

[4] Bukar M, Audu BM, Yahaya UR, et al. Anaemia in pregnancy at booking in Gombe, North-eastern Nigeria. J Obstet Gynaecol 2008;28(8):775-8.

[5] Levy A, Fraser D, Katz M, et al. Maternal anemia during pregnancy is an independent risk factor for low birthweight and preterm delivery. Eur J Obstet Gynecol Reprod Biol 2005;122(2):182-6.

[6] Brabin BJ, Hakimi M, Pelletier D. An analysis of anemia and pregnancy-related maternal mortality. J Nutr 2001;131(2S-2):604S-15S.

[7] McFee JG. Anemia in pregnancy-a reappraisal. Obstet Gynecol Surv 1973;28(11):769-93.

[8] Ogbeide O, Wagbatsoma V, Orhue A. Anaemia in pregnancy. East Afr Med J 1994;71(10):671-3.
[9] https://www.msdmanuals.com/enin/professional/gynecology-and-obstetrics/pregnancycomplicated-by-disease/anemia-in-pregnancy

[10] Sifakis S, Pharmakides G. Anemia in pregnancy. Ann N Y Acad Sci 2000;900(1):125-36.

[11] van den Broek NR, Letsky EA. Etiology of anemia in pregnancy in south Malawi. Am J Clin Nutr 2000;72(1 Suppl):247S-56S.

[12] Balasubramanian T, Aravazhi M, Sampath SD. Awareness of anaemia among pregnant women and impact of demographic factors on their haemoglobin status. International Journal of Scientific Study 2016;3(12):3035.

[13] Patra S, Pasrija S, Trivedi SS, et al. Maternal and perinatal outcome in patients with severe anemia in pregnancy. Int J Gynecol Obstet 2005;91(2):164-5.

[14] Dwumfour-Asare B, Kwapong MA. Anaemia awareness, beliefs and practices among pregnant women: a baseline assessment at Brosankro community in Ghana. Journal of Natural Sciences Research 2013;3(15). 